Gustavo Guerrero, Jorge J. Locane, Benjamin Loy, Gesine Müller

\title{
A modo de introducción. Literatura latinoamericana: inflexiones de un término
}

La literatura latinoamericana es un constructo cuya gestación se puede fechar en los años 60 del siglo pasado. Después de la Revolución Cubana, el territorio al sur del Río Bravo termina por recortarse y ganar visibilidad para el resto del mundo como una unidad geocultural en efecto existente y más o menos homogénea. Si se le da crédito a los postulados de José Donoso en su Historia personal del "boom" (1972), es en estos años que la literatura latinoamericana comienza a proyectarse programáticamente y con altura hacia el mundo, y a ser percibida como expresión de "grandes símbolos identitarios" asociados con la región (Avelar 23; cf. también Müller). Pero también habría que anotar que, como efecto de la Revolución Cubana, por aquel entonces se acentuó en América Latina un esfuerzo crítico por aislar -o, acaso, inventar- las especificidades que harían de la literatura del subcontinente algo relativamente homogéneo y diferenciado de las literaturas de otras regiones del mundo. Junto con este proceso vernáculo, además, habría que mencionar la propagación de los estudios areales en los centros de poder mundial comprometidos en la así denominada Guerra Fría. Varias publicaciones de la época, cuyo fundamento suele retrotraerse hasta "Nuestra América” (1891), de José Martí, son testimonio del Zeitgeist que dominaba la región en dicha coyuntura. La literatura latinoamericana como proceso (1985), una de las varias compilaciones de Ana Pizarro; La novela en América Latina (1982), de Ángel Rama; Literatura y conciencia política en América Latina (1969), de Alejo Carpentier; o América Latina en su literatura (1972), editado por César Fernández Moreno y financiado por la UNESCO, valen como ejemplos.

De este último, que surgió de un encuentro y debate entre algunos de los críticos más reconocidos del momento en San José de Costa Rica en 1968, interesa un pasaje de la "Introducción”, redactada por César Fernández Moreno, que condensa varios de los factores mencionados arriba y permite ilustrar de manera gráfica cuál era espíritu de la época. Dice así:

el mundo contemporáneo redescubre con nuevo deslumbramiento este complejo que insiste en llamarse América Latina, entidad todavía no definida, pero que presenta a simple vista la consistencia de lo real. Si profundizáramos en busca de las raíces de esta ostensible unidad, su historia suministra esta primera nota: sucesiva dependencia del conjunto respecto de una potencia exterior. [...]

Ә Open Access. () 2020 Gustavo Guerrero et al., published by De Gruyter. (cc) By-NC-ND This work is licensed under a Creative Commons Attribution-NonCommercial-NoDerivatives 4.0 International License.

https://doi.org/10.1515/9783110673678-001 
Esta nota de dependencia sería, acaso, la primera a considerar para determinar el fugitivo concepto de América Latina. Y, la segunda, su inmersión en la más fuerte polaridad histórica de la actualidad: el abismo que se abre entre los países ricos y los pobres; oposición más vasta que la anterior, pero no contradictoria con ella, ya que se ilustra en el conjunto de las Américas, donde la anglosajona es la rica y la latina es la pobre. Estos dos criterios se complementan y confirman por un tercero más elemental: el geográfico, en que se apoyan, expresa o tácitamente, todos los que hasta ahora hemos compulsado. América Latina sería toda aquella tierra americana que queda al sur del río Grande o Bravo (que marca el límite de Estados Unidos con México). La habitualidad de esta expresión (al sur del río Grande, o Bravo) sería prueba de su veracidad: al sur de este río existe cierta homogeneidad cultural, política, social, lingüística, religiosa. (9)

Y unas páginas más adelante, agrega que "El rumbo no podía ser más acertado: los escritores de esta región, por así decirlo, no tienen más remedio que expresar el mundo que los circunda y se les impone, creciente y bullente, mundo de contradicciones y desgarramientos, de contemplación y acción aniquiladoras” (13). Así, para uno de los observadores de la época, la literatura latinoamericana se manifestaba signada por el estigma de una opresión geopolítica histórica anclada a una geografía concreta y claramente delimitada. "Los escritores de la región", sostenía Fernández Moreno, estarían destinados a dar cuenta de la "realidad" distintiva de América Latina.

En el mismo contexto y bajo el mismo signo ontológico latinoamericanista, hizo sus contribuciones Roberto Fernández Retamar. En su ensayo de 1975 "Para una teoría de la literatura hispanoamericana” argumentaba que, puesto que el mundo lejos está de ser homogéneo, no sería posible identificar una literatura mundial y que, por tal razón, la crítica latinoamericana debía fundar convenciones de abordaje específicas para lo que él consideraba su objeto natural:

Las teorías de la literatura hispanoamericana, pues, no podrían forjarse trasladándole e imponiéndole en bloque criterios que fueron forjados en relación con otras literaturas, las literaturas metropolitanas. Tales criterios, como sabemos, han sido propuestos -e introyectados por nosotros- como de validez universal. Pero también sabemos que ello, en conjunto, es falso, y no representa sino otra manifestación del colonialismo cultural que hemos sufrido, y no hemos dejado enteramente de sufrir, como secuela del colonialismo político y económico. Frente a esa seudouniversalidad, tenemos que proclamar la simple y necesaria verdad de que una teoría de la literatura es la teoría de una literatura. (82)

De modo que, durante el período -en lo que va de mediados de siglo hasta la Caída del Muro de Berlín-, cierta crítica cultural se dio a la tarea de establecer criterios geográficos, políticos y culturales para delimitar América Latina, de identificar rasgos distintivos para su literatura $y$, finalmente, también de exhibir ante el mundo ese objeto como patrimonio singular y bien de consumo con algunas trazas de exotismo. Esta labor crítica correspondía al discurso sostenido por 
el grupo de escritores que bajo el lema del boom se constituyeron en los creadores literarios principales de estas operaciones identitarias y mercantiles. La fórmula de una nueva unidad latinoamericana en términos literario-culturales inauguraba un nuevo discurso universalista nutrido por la posibilidad "de hablar con rigor de la contemporaneidad del escritor latinoamericano, quien súbitamente es parte de un presente cultural común: [. . . ] nuestros escritores pueden dirigir sus preguntas no sólo al presente latinoamericano sino también a un futuro que, cada vez más, también será común al nivel de la cultura y de la condición espiritual de todos los hombres" (Fuentes 35). Frente a un presunto agotamiento de la literatura europea y su poder denominador en tanto "meridiano de Greenwich" de la república mundial de las letras, para usar el término de Pascale Casanova, la literatura latinoamericana se inscribe con una fuerza renovada en el campo literario global formulando de manera ostentativa su nuevo estatus, como cuando Mario Vargas Llosa afirma que "cuando tenían a Proust y Joyce, los europeos se interesaban apenas o nada por Santos Chocano o Eustasio Rivera. Pero ahora que sólo tienen a Robbe-Grillet, Nathalie Sarraute o Giorgio Bassani, ¿cómo no volverán los ojos fuera de sus fronteras en busca de escritores más interesantes, menos letárgicos y más vivos?” (cit. en Avelar 45) ${ }^{1}$.

Con la Caída del Muro, el desmoronamiento de los proyectos comunistas y la fractura del orden bipolar, no obstante, el armado latinoamericanista comienza a resquebrajarse. Sucede que, en la nueva fase de la globalización, al mismo tiempo que algunas instituciones y organismos privados logran ganar cobertura global, los flujos económicos y culturales entre las diferentes regiones del mundo se incentivan a un nivel sin precedentes. Mediante mecanismos como el Consenso de Washington, el proyecto neoliberal se consolida a escala mundial y transforma, aceleradamente, los paisajes culturales al punto de que la singularidad y los recortes identitarios se difuminan y pierden sustento o, como anota María Eugenia Mudrovcic, “si en los '70 'todo era política', a partir de los '90 la economía del mercado pasa a ser el organizador transnacional de la cultura. Al cambiar las reglas de circulación y gestión cultural con miras a lograr una rápida escalada en

1 Al mismo tiempo y más allá de la exhibición de ese optimismo se encuentran, sin embargo, también posiciones más críticas como cuando Julio Cortázar, en una de sus cartas, formula severas dudas con respecto a la profundidad y perdurabilidad de la nueva presencia internacional de la región al sostener que "la opinión corriente sobre nuestra influencia en su literatura me parece más un deseo que una realidad; no basta un boom, no bastan tan pocas décadas para incidir decisivamente en estructuras que para bien y para mal se cierran sobre sí mismas a la vez que coquetean con los recién llegados, vengan de Europa oriental, del Japón, de Estados Unidos, de Australia o de América Latina. Los europeos dialogan ya con nosotros en el plano literario, pero de alguna manera lo hacen todavía acariciándonos la cabeza como cuando se le habla a un niño" (284-285). 
la rentabilidad y la eficiencia, el nuevo modelo de desarrollo económico cambia también el lugar que ocupa la cultura en la sociedad" (756). Los emblemas del capitalismo cruzan fronteras, colonizan la apariencia urbana en todo el mundo y dan paso a procesos de subjetivación posnacionales y posidentitarios. El mundo, así, se comprime y adquiere una apariencia relativamente uniforme, hilvanada por el discurso del capitalismo global. El mundo -ahora sí- puede ser considerado uno, y, en consecuencia, los recortes que se habían aplicado a la literatura pierden credibilidad. En 1999 aparece el ya citado La république mondiale des lettres, de Casanova, y, con él, queda inaugurado oficialmente el hoy ya frondoso debate sobre literatura mundial que tiende a reconsiderar los fenómenos literarios que habían sido adjetivados con algún gentilicio particularista como parte de un sistema global.

Desde entonces, en lo que respecta más concretamente a América Latina, tanto una zona importante de la crítica literaria especializada como un grupo nutrido de escritores con presencia y voz en el mercado internacional van a comenzar a borronear los contornos que las operaciones críticas de las décadas anteriores habían delineado para las literaturas vernáculas. Así, ese corpus, que había sido minuciosa y exitosamente construido, va a tender a diluirse en el corpus mayor de la literatura mundial. Sin atributos distintivos no hay objeto; y, si no hay objeto, tampoco puede haber estudios especializados. Estamos en el reino de McOndo y de El crack, y también en el de algunos estudios literarios que creen ver en la globalización cultural una herramienta para combatir los vetustos nacionalismos y las proclamas nativistas.

Así, en 1999, Carlos Cortés publica el artículo "La literatura latinoamericana (ya) no existe”. En el 2003 el grupo Planeta reúne un grupo de escritores en Sevilla donde se suceden los alegatos cosmopolitas y las descalificaciones de cualquier esencialismo latinoamericanista. En el volumen que reúne las intervenciones, $\mathrm{Pa}$ labra de América, del 2004, aparece la contribución de Volpi "El fin de la narrativa latinoamericana" que se prolongará en el posterior libro El insomnio de Bolivar (2009). Del 2007 es "Y finalmente, ¿existe una literatura latinoamericana?", de Jorge Fornet, formulado en la misma sintonía. También se podrían mencionar textos de Gioconda Marún o Catalina Quesada Gómez. ${ }^{2}$

En "Crítica del panorama” (2009), Gustavo Guerrero recorre algunas de estas intervenciones previas y anota:

2 Habría que aclarar que, si bien la mayoría de estos aportes intenta reinscribir la producción literaria latinoamericana en el marco mayor de la literatura mundial, algunos apuntan más bien a señalar la atomización, la dispersión y las relocalizaciones. 
el fin de los metarrelatos modernos no sólo ha llevado en América Latina a [una] irreprensible diversificación de la producción literaria y las maneras de leerla. Al suscitar vivas tensiones entre las identidades locales y globales, ha dado pie también a un cuestionamiento del concepto mismo de literatura latinoamericana en tanto categoría supranacional y denominador común. $(26)^{3}$

El acta de defunción ha sido, pues, expedida, y los modelos teóricos que habían sido elaborados en el mundo fragmentado anterior a la Caída del Muro para explicar las literaturas regionales parecieran ahora definitivamente incapaces de dar cuenta de los productos culturales elaborados en el marco del actual diseño global.

Este libro intenta reflexionar sobre las tensiones que se dan en los procesos de inscripción de lo que fue delimitado como literatura latinoamericana en dinámicas de circulación internacional. Sin dar necesariamente por supuesto que la literatura latinoamericana, en tanto construcción, se haya desintegrado en el corpus mayor de la literatura mundial, procura examinar mecanismos mediante los cuales parte de la primera se abre paso e integra en la segunda. La literatura latinoamericana mundial sería, pues, una que se halla en tránsito entre un dominio y el otro, sería la literatura que con algún tipo de impronta identificable como latinoamericana encuentra una recepción en horizontes de mayor o menor alcance global ${ }^{4}$. Antes que celebrar o enjuiciar el proceso de proyección hacia el mundo, este volumen intenta analizar los dispositivos que lo propulsan y lo convierten en algo en efecto posible. Interesa, ante todo, la traducción y también los gatekeepers, es decir, los mediadores con poder suficiente como para abrir canales de circulación en el dominio internacional (cf. Marling). Sin traducción -con todo lo que ella implica en términos de pérdida y de ganancia, con sus límites y posibilidades- y sin gatekeepers, lo que incluye determinadas "instituciones" (cf. Helgesson/Vermeulen), no hay literatura mundial. Pero también interesa el negativo de la literatura latinoamericana mundial, esto es, las literaturas que presentan fuertes resistencias al ingreso en flujos internacionales o que permanecen enraizadas en configuraciones geoculturales del continente y que, por tal razón y porque no imperiosamente

3 Véase también su Paisajes en movimiento (2018).

4 Mayores precisiones sobre el término se pueden encontrar en las monografías Beyond Bolaño (2015), de Héctor Hoyos, Strategic Occidentalism (2018), de Ignacio M. Sánchez Prado, quien examina la "literatura mexicana mundial", y De la literatura latinoamericana a la literatura (latinoamericana) mundial (2019), de Jorge J. Locane. El cambio de enfoque hacia nuevas relaciones globales de la literatura latinoamericana se expresa en publicaciones recientes como Re-mapping World Literature. Writing, Book Markets and Epistemologies between Latin America and the Global South (2018), editado por Gesine Müller, Jorge J. Locane y Benjamin Loy. 
responden a horizontes de recepción nacionales, pueden ser denominadas literaturas locales. Estos son, pues, los tres articuladores que, respectivamente, organizan este libro: 1. gatekeepers, 2. traducción y 3. literaturas locales.

Con contribuciones de Gesine Müller, Judith Illerhaus, Marteen Steenmeijer, Gustavo Guerrero, Ana Gallego Cuiñas e Idun Heir Senstad, el primer bloque examina el accionar de diferentes gatekeepers -editores, traductores, premios y festivales- en espacios nacionales de recepción como Alemania, Holanda, Francia y Noruega, en lo que va de los años 60, cuando la literatura latinoamericana adquiere estatus orgánico y delimitado, hasta el presente, cuando, al menos en algunos de sus segmentos, comienza a desdibujarse como un componente más de la literatura mundial. El artículo de Müller, "Gatekeeping and the Conceptualization of World Literature in the Publishing Industry. The Case of Siegfried Unseld and Octavio Paz", se centra en la labor de Siegfried Unseld, en tanto editor general de la editorial Suhrkamp, para la inserción de Octavio Paz en el mercado germanohablante desde fines de los años 70. De acuerdo con sus argumentos, Unseld habría elaborado una imagen de Paz que permitía asimilarlo tanto a los postulados ideológicos de una izquierda europea no dogmática como a fórmulas que le asignan al sistema sociocultural latinoamericano un carácter exótico y lo convierten, según palabras de Unseld, en una suerte de far west. Por su parte, Illerhaus, con la contribución "Premios porteros. La función del Premio Biblioteca Breve con base en números”, enfoca la atención en el impacto del premio otorgado por la casa editorial Seix Barral en la producción de literatura latinoamericana mundial. Su estudio ensaya un examen comparativo entre las dos etapas del premio, la de los años 60 y la que se inaugura a fines de los 90, a partir de indicadores que informan acerca de la cantidad y celeridad de las traducciones de los títulos reconocidos con el galardón. El texto de Steenmeijer, "Ascenso y ocaso de una marca: la literatura hispanoamericana en Holanda”, ofrece un recorrido por la recepción de la literatura latinoamericana en los Países Bajos durante los últimos cincuenta años, precisamente, como "marca” que se inaugura con la figura de Jorge Luis Borges y con el boom para, luego, volver a perder ese estatus. A través de un análisis de numerosos paratextos, críticas de prensa o la caracterización en festivales de literatura, Steenmeijer retrata, con base en ejemplos tan diferentes como Isabel Allende, Roberto Bolaño o César Aira, el progresivo proceso de disolución del consenso sobre las pautas que servían de marco para los escritores latinoamericanos en busca de un público holandés, hecho que interpreta como una especie de "descontinentalización” de la literatura latinoamericana y una forma específica de conversión en "literatura mundial”. En su aporte "José Lezama Lima en Francia: apuntes sobre la mediación editorial, la traducción y la recepción de Paradiso”, Guerrero examina el complejo proceso que, por mediación de Julio Cortázar y Severo Sarduy, 
condujo a la publicación de la novela de José Lezama Lima en Francia en 1971. En el contexto de la Guerra Fría, la publicación de Paradiso no estuvo exenta te tensiones determinadas por la política internacional y también por las dificultades técnicas que condujo a un relevo en el trabajo de traducción, primero, encargado a Claude Couffon y, luego, a Didier Coste. No obstante estas "interferencias", que informan sobre los numerosos factores que afectan la producción de literatura mundial, Paradiso fue publicada por Seuil con un significativo éxito de ventas que, a su vez, se tradujo en la consagración de la editorial y en la posterior traducción de la novela a otras lenguas hegemónicas. Gallego Cuiñas, con su artículo "Feminismo y literatura (argentina) mundial: Selva Almada, Mariana Enríquez y Samanta Schweblin”, argumenta que uno de los grandes recortes que permite pensar la actual propagación de literatura argentina por el mundo estaría delimitado por la impronta femenina. De acuerdo con sus planteos, escritoras como Almada, Enríquez y Schweblin habrían pasado por un proceso de reconocimiento nacional, fundado en la plataforma de asignación de valor simbólico que constituye la red local de editoriales independientes, para, luego, insertarse exitosamente en los catálogos de la industria literaria transnacional. Su conclusión es que la literatura mundial se apropia de estos nuevos modos de significar la experiencia femenina convirtiéndolos "en una mercancía feminista", exportable en virtud de un castellano neutro, de la apelación a subjetividades globales y a una poética general no contrahegemónica -decolonial- sino, antes, confirmatoria del orden mundial. Por último, con "Literary Diversity through Translation: The Role of Independent Cultural Mediators in the Selection of Cuban Literature in Norway", Senstad examina el papel de los traductores, presentados como mediadores culturales independientes, en la selección de la literatura cubana traducida al noruego entre 1990 y 2014. Explora cómo y por qué estos sugieren títulos a los editores y cómo, a través de sus elecciones, contribuyen a la diversidad literaria. Los editores, argumenta Senstad, son quienes tienen la última palabra, ya que son ellos quienes toman la decisión final sobre si encargar o no una traducción; sin embargo, sus decisiones siempre están integradas en redes transnacionales de determinación más amplias que incluyen a los traductores. Su conclusión es que, frente a la selección determinada más directamente por editores y agentes, centrada en textos escritos en inglés por escritores de la diáspora cubana, los traductores abogaron por e introdujeron literatura cubana canonizada del siglo XX o textos de escritores cubanos contemporáneos con residencia en Cuba, originalmente escritos en español.

El bloque dedicado a la traducción incluye artículos de Annick Louis, Liset Díaz Martínez, Yehua Chen, Sara Carini, Ilse Logie, Cecilia Alvstad y Benjamin Loy. También acá se examinan diferentes itinerarios y coyunturas: desde la 
temprana traducción de Jorge Luis Borges al francés y la de Mario Vargas Llosa al chino mandarín hasta la de las nuevas generaciones de escritores, como la de Valeria Luiselli al inglés y la de Yuri Herrera al noruego. Lejos de abordar la traducción a partir de la premisa de la transparencia, estos estudios la piensan como dificultad y desafío. Tratan de analizar sus efectos secundarios y sus límites: los desplazamientos, las asperezas y lo intransferible. Otra línea de análisis que exploran es la que refiere a la tensión entre la literatura más aferrada a formas verbales vernáculas y, por lo tanto, resistente a la circulación internacional vía traducción y la que, desde su origen, se la concibe orientada al mercado internacional o, vale decir, pretraducida. El artículo de Louis, "El Aleph de Roger Caillois en Gallimard o de cómo salir del laberinto”, examina el volumen Labyrinthes, atribuido a Borges y publicado y traducido por Roger Caillois en 1953 como volumen inaugural de la colección "La Cruz del Sur” de la editorial Gallimard. Según informa Louis, este volumen es una fabricación de Caillois, que responde a su lectura de la obra de Borges presentada en el prefacio. El laberinto como supuesto tema fundamental del universo borgeano se impone a partir de esta publicación, que, a su vez, va a dar lugar a numerosas traducciones a diferentes idiomas (inglés y alemán, en particular). Esta operación crítica de traducción, cuyo éxito resulta evidente, no solo construye un Borges para el mundo, sensiblemente distinto del que circula en versión original, sino que también tendrá el efecto inesperado de atrasar la publicación del libro El Aleph, que va a aparecer en Francia recién en 1967. El texto de Díaz Martínez, “Gabriel García Márquez traducido: observaciones sobre la variación”, desarrolla un estudio comparativo de las traducciones al francés del escritor colombiano desde un enfoque lexicológico. Focalizándose en la variación diatópica y en el nivel léxico, propone un estudio interdisciplinario de la realidad lingüística de Colombia plasmada en textos de García Márquez que demuestra que las versiones francesas difícilmente presentan valores equivalentes en relación con la variedad contenida en los textos fuente. Estos fenómenos -que, desde luego, pueden reclamar una representatividad dentro de los complejos procesos de traducción en la literatura latinoamericana mundial- adquieren una importancia particular en su tendencia a reducir sistemáticamente la riqueza léxico-cultural que un autor como García Márquez vuelca en su escritura y que ha sido atendida por los traductores franceses solamente de manera muy parcial. Chen, por su parte, en su contribución titulada "Traitors or Traders? A Brief Analysis of Chinese Translations of Latin American Boom Writers", ofrece un close reading de las traducciones al chino mandarín de textos del boom como Conversación en La Catedral, de Mario Vargas Llosa, y El lugar sin límites, de José Donoso. A partir de la observación de que diferentes traductores chinos, a pesar de que poseían un bagaje similar y de que publicaron sus 
textos en un mismo contexto histórico, desplegaron diferentes estrategias de traducción para conseguir un cierto estilo narrativo, Chen sostiene que lo que está en juego en el acto de traducir son, antes, decisiones que "errores" o "malas interpretaciones" de los textos fuente. Concluye, finalmente, con la idea de que, puesto que, detrás de cada traducción, hay una persona viva que trabaja a conciencia durante largos períodos, y que, detrás de cada elección, hay una razón, no sería adecuado equiparar la práctica con la "traición”. Carini, a su vez, con su artículo “"Tante cose venivano pubblicate ma hanno dovuto aspettare un altro momento': la literatura latinoamericana y sus re-traducciones”, dirige la atención a las nuevas traducciones de literatura latinoamericana que fueron publicadas en Italia desde el año 2000 en adelante. El estudio se detiene, particularmente, en los casos de Paradiso, de Lezama Lima, y Farabeuf, de Salvador Elizondo, para examinar los cambios que ha habido entre la primera salida al mercado de ambas novelas y su nueva versión. Carini subraya cuáles han sido las modificaciones de las perspectivas de la crítica, pero también cuáles han sido las palabras clave utilizadas por las editoriales para difundir y presentar estas obras al público. Este procedimiento le permite a Carini identificar qué clima y qué interés subyace a la recepción de la literatura latinoamericana hoy con respecto a los años 60 y 70 y cómo se filtra la literatura latinoamericana en esta nueva fase de difusión en la que, según argumenta, la escritura de Roberto Bolaño domina el escenario literario tanto por éxito de público como de crítica. El estudio de Logie, “¿Escritos en la traducción y para la traducción? Dos ejemplos: Valeria Luiselli y Mario Bellatin”, retoma los planteos de Rebecca Walkowitz, en su libro Born Translated (2015), para examinar Los ingrávidos, de Valeria Luiselli, y Jacobo el mutante, de Mario Bellatin. Según propone, el carácter translaticio de estos textos se prolonga en sus traducciones, lo que se manifiesta en algunos cambios notables en las traducciones al inglés de ambos. Pero la traducción, sostiene a continuación, puede llegar a generar algo nuevo, una especie de textualidad expandida. Así, a raíz de una carta que recibió de su traductor inglés, Bellatin escribió una nueva versión de su texto, Jacobo reloaded, que posteriormente llegó a ser la fuente de Jacob the Mutant. Logie concluye con una reflexión sobre la dificultad para evaluar este tipo de literatura ya que, según sus detractores (como Tim Parks o Brian Lennon), la circulación global y la preocupación por la traducibilidad llevan a una creciente homogeneidad, mientras que otros estudiosos (como Walkowitz) consideran que se trata de una nueva modalidad de difusión que no está reñida con una crítica a la industria editorial transnacional. La contribución de Alvstad, “Anthropology over Aesthetics: On the Poetics of Movement and Multilingualism in Three Translations of Yuri Herrera's Señales que precederán al fin del mundo", ofrece un estudio comparativo de traducciones al 
noruego, al sueco y al inglés de la novela del escritor mexicano. Según argumenta, la literatura que suele ingresar en el mercado de los países nórdicos enfatiza información antropológica antes que el valor literario. La literatura de las regiones del sur del mundo que, bajo estas premisas se difunde en los países nórdicos, es la que suele ser caracterizada como "literatura mundial”. El análisis minucioso de las traducciones, sin considerar la información paratextual, le permite proponer que varios dispositivos poéticos del original, como los neologismos y los silencios léxicos, que rodean los temas del movimiento y el multilingüismo, fueron burlados en las traducciones. Desde el punto de vista técnico, sostiene, los traductores normalizaron, explicitaron y simplificaron, es decir, crearon características lingüísticas en las traducciones que respetan patrones de recepción estandarizados. Alvstad concluye que los efectos de estos procesos de normalización, explicitación y simplificación debilitan importantes capas estéticas del texto de Herrera, a la vez que refuerzan el marco antropológico asociado con México, Estados Unidos y la frontera entre ambos países. La contribución de Loy que cierra el bloque, "Glotopolíticas literarias entre resistencia y mercado: Bolaño en traducción, la traducción en Bolaño”, también ofrece un estudio comparativo de diferentes traducciones. El artículo parte de una crítica de un aspecto paradójico en cuanto al estatus de la traducción en el contexto de los debates teóricos actuales sobre una literatura mundial: si bien la traducción es valorada porque el acto de traducir como tal forma parte del discurso y de las prácticas cosmopolitas contemporáneos, la gran mayoría de los estudios dedicados al tema -observa Loy- no indagan realmente en las dimensiones concretas de las traducciones en cuanto práctica translaticia. Ante ese panorama, elabora un estudio de caso a partir de la obra de Bolaño y el análisis comparativo de las traducciones de su novela Estrella distante al inglés, alemán y francés. El estudio sostiene la paradójica hipótesis de que, por una parte, la idea de la traducción y de la traducibilidad es fundamental en la obra de Bolaño, pero que, por el otro, posee solamente una importancia secundaria para la obra en cuanto texto traducido en circulación internacional y como parte de una literatura mundial contemporánea.

El tercer y último bloque reúne los aportes de Ignacio Sánchez M. Prado, Silja Helber, Matías Moscardi y Jorge J. Locane. Con un recorte temporal más circunscripto al presente y al marco general de la actual fase de la globalización, estos estudios abordan proyectos, ya sea editoriales o literarios, que de diferentes modos interfieren la lógica de la literatura latinoamericana mundial y se mantienen arraigados a dinámicas de circulación y recepción locales. Ante un panorama de publicación, circulación y consumo de la literatura latinoamericana que ha sido objeto de transformaciones infraestructurales profundas, el artículo de 
Sánchez Prado, “Cosmopolitismo copyleft. Tumbona Ediciones, autonomía y localidad”, examina el rol de las literaturas locales como resistencia a la hegemonización de la "literatura mundial", poniendo especial atención a la materialidad infraestructural de la producción literaria, y la relación tensional y paradójica de la forma literaria respecto a esa materialidad. Sánchez Prado reflexiona sobre este problema a partir del proyecto Tumbona Ediciones, una editorial independiente fundada en principios de autonomía, colectivismo y copyleft en México en 2005, y sus estrategias de construcción de una literatura mundial desde abajo, desde espacios alternativos y a contrapelo de la literatura mundial impuesta desde arriba a través de circuitos hegemónicos de circulación transnacional. El desarrollo de sus observaciones se asienta en una acepción particular del concepto de "hospitalidad" (acuñado por Jacques Derrida) que, según sostiene, se encuentra realizado en la política y el catálogo editorial de Tumbona como una lógica de la circulación que abre, incondicionalmente, las puertas de una tradición a una serie de libros y bienes culturales mundiales y que posee una dialéctica específica: abre un espacio para dar la bienvenida a escrituras nómadas que no entrarían de manera clara en la tradición mexicana, y a una tradición mexicana cuya relación material con el mundo y la literatura sale de los parámetros de los cosmopolitismos hegemónicos, tanto del mercado como del campo de producción cultural del siglo XX. En su artículo “¿Cuanto más marginal, más central? - La escritura de Rita Indiana”, Helber analiza la trayectoria literaria de la figura polifacética que representa la escritora dominicana Rita Indiana, concediendo prioridad a su entrada en el campo literario, primero, nacional y, luego, internacional por la publicación en una editorial española y la traducción al inglés de su novela Papi. Mediante un breve análisis de esta última, Helber indaga si con la inserción en un campo editorial transnacional Indiana deja de formar parte de la "escritura local” y se convierte en lo que se podría llamar literatura con potencial exportable. Como conclusión sostiene que, aunque Papi bien puede ser caracterizada como literatura local, se presta también para una lectura, recepción y circulación a nivel global, y que, por consiguiente, Indiana no es una escritora mundial, en el sentido más estricto, sino una escritora glocal, ya que conecta y reúne temas y elementos globales y locales en su escritura. La pregunta por el género literario y en cómo esta variable afecta la circulación (internacional) es de central importancia tanto en la contribución de Sánchez Prado como en la de Moscardi, “Audiometrías contemporáneas. La escucha como modo de circulación en la poesía argentina actual”. En su artículo, Moscardi examina la puesta en voz de la poesía argentina actual en tanto práctica poética que desborda o elude el formato del libro como soporte privilegiado -y con ello la industria editorial-, para intervenir más allá de él y de sus canales de circulación 
concomitantes. De acuerdo con sus argumentos, en el corpus analizado, de Alfonsina Brión, de Milton López, no parecería existir un "legible" sin un "audible" asociado. De esta manera, concluye, la voz y la escucha parecieran imponer nuevos desafíos para la circulación, la recepción y el abordaje de los textos poéticos; constituirían, además, una dimensión de la literatura intransferible al circuito internacional, pero, en contrapartida, capaz de producir enlaces y generar comunidad a escala local. Como cierre, el texto de Locane, "Los últimos centímetros de un lápiz de grafito’. Mercado, (neo)autonomía y la ‘operación Mike Wilson’”, propone, a partir de una revisión crítica del término "posautonomía” de Josefina Ludmer, un examen de estrategias editoriales autogestivas que cuestionan la lógica de gestión de la industria editorial transnacional y su régimen de heteronomía. Junto con el examen de una imagen extraída de Leñador o ruinas continentales, de Wilson, donde un personaje produce escritura fuera de todo marco posible de recepción, Locane desarrolla una lectura del acto concreto de Wilson de haber autopublicado, después de haber incursionado en el circuito de la literatura mundial, un relato en formato booklet que se entregaba gratuitamente en algunas librerías de Santiago de Chile. Como conclusión, sostiene que esta reinscripción del artefacto literario en un régimen de (neo)autonomía supondría un necesario desprendimiento del circuito internacional y de la literatura mundial: un rechazo de la industria transnacional para pasar a la edición independiente radical o, más aún, a la autoedición.

Hecha esta introducción, esperamos que el volumen acá presentado encuentre sus lectores y contribuya a enriquecer los actuales debates sobre literatura latinoamericana, literatura mundial y globalización. Por lo demás, no queremos cerrar esta introducción sin hacer público nuestro agradecimiento al European Research Council por el apoyo económico a nuestras actividades de investigación y el financiamiento de esta publicación.

\section{Bibliografía}

Avelar, Idelber. Alegorías de la derrota: la ficción postdictatorial y el trabajo del duelo. Cuarto propio, 2000.

Bolaño, Roberto et al. Palabra de América. Seix Barral, 2004.

Carpentier, Alejo. Literatura y conciencia política en América Latina. Alberto Corazón Editor, 1969.

Casanova, Pascale. La republique mondiale des lettres. Seuil, 1999.

Cortázar, Julio. Obra crítica 3. Ed. Saúl Sosnowski. Alfaguara, 1994.

Cortés, Carlos. "La literatura latinoamericana (ya) no existe”. Cuadernos Hispanoamericanos, 592, 1999, pp. 59-67.

Donoso, José. Historia personal del “boom”. Anagrama, 1972. 
Fernández Moreno, César (coord.). América Latina en su literatura. Siglo XXI/UNESCO, 1972.

---. "Introducción”. América Latina en su literatura. Coord. César Fernández Moreno. Siglo XXI/ UNESCO, 1972, pp. 5-18.

Fernández Retamar, Roberto. "Para una teoría de la literatura hispanoamericana”. Para una teoría de la literatura hispanoamericana. Publicaciones del Instituto Caro y Cuervo, 1995 [1975], pp. 74-87.

Fornet, Jorge. "Y finalmente, ¿existe una literatura latinoamericana?”. La jiribilla. Revista de cultura cubana, 9-15 de junio 2007. http://epoca2.lajiribilla.cu/2007/n318_06/318_01. html, consultado 1 de marzo 2019.

Fuentes, Carlos. La nueva novela latinoamericana. Joaquín Mortiz, 1969.

Guerrero, Gustavo. Paisajes en movimiento. Literatura y cambio social entre dos siglos. Eterna Cadencia, 2018.

Guerrero, Gustavo. “Crítica del panorama”. Letras libres, 93, 2009, pp. 24-28.

Helgesson, Stefan/Pieter Vermeulen (eds.). Institutions of World Literature: Writing, Translation, Markets. Routledge, 2016.

Hoyos, Héctor. Beyond Bolaño. The Global Latin American Novel. Columbia University Press, 2015.

Locane, Jorge J. De la literatura latinoamericana a la literatura (latinoamericana) mundial. Condiciones materiales, procesos y actores. De Gruyter, 2019.

Marling, William. Gatekeepers. The Emergence of World Literature \& the 1960s.

Oxford University Press, 2016.

Marún, Gioconda. Latinoamérica y la literatura mundial. Dunken, 2013.

Mudrovcic, María Eugenia. "Políticas culturales en los procesos de integración regional: el sector editorial en el Mercosur”. Revista Iberoamericana LXVII, 197, 2001, pp. 755-766.

Müller, Gesine. Die Boom-Autoren heute: García Márquez, Fuentes, Vargas Llosa, Donoso und ihr Abschied von den großen identitätsstiftenden Entwürfen. Vervuert, 2004.

Müller, Gesine /Jorge J. Locane/Benjamin Loy (eds.). Re-mapping World Literature. Writing, Book Markets, and Epistemologies between Latin America and the Global South. De Gruyter, 2018.

Pizarro, Ana (coord.). La literatura latinoamericana como proceso. Centro Editor de América Latina, 1985.

Quesada Gómez, Catalina. Literatura y globalización: la narrativa hispanoamericana en el siglo XXI (espacio, tiempos y géneros). Universidad de Antioquia, 2014.

Rama, Ángel. La novela en América Latina. Panoramas 1920-1980. Colcultura, 1982.

Sánchez Prado, Ignacio M. Strategic Occidentalism. On Mexican Fiction, the Neoliberal Book Market, and the Question of World Literature. Northwestern University Press, 2018.

Volpi, Jorge. El insomnio de Bolivar. Cuatro consideraciones intempestivas sobre América Latina en el siglo XXI. Debate, 2009.

---. "El fin de la narrativa latinoamericana". Palabra de América. Roberto Bolaño et al. Seix Barral, 2004, pp. 206-223. 
